\title{
Global Tracking and Disturbance Rejection of a Class of Lower Triangular Systems Subject to an Unknown Exosystem
}

\author{
Zhiyong Chen, Lu Liu and Jie Huang
}

\begin{abstract}
In this paper, we study the asymptotic tracking and disturbance rejection problem for a class of uncertain nonlinear lower triangular systems subject to an uncertain exosystem. The same problem was studied before for the same class of systems with the objective of asymptotic tracking only. By utilizing a newly developed robust adaptive controller design approach, we are able to simultaneously handle the objectives of disturbance rejection and asymptotic tracking.

Index Terms-Robust control, Adaptive control, Internal model, Nonlinear systems, Regulation, Stabilization
\end{abstract}

\section{INTRODUCTION}

In this paper, we study the asymptotic tracking and disturbance rejection problem for a class of uncertain nonlinear lower triangular systems. Both reference inputs and disturbances are generated by a linear autonomous differential equation called exosystem. The problem is also called output regulation problem or servomechanism problem. The problem has been extensively studied for the case where the exosystem is exactly known [1], [2], [3], [4], [5], [6], etc. When the exosystem contains uncertainty, the problem has also been studied for various systems in, e.g., [3], [7], and [8]. In particular, a global asymptotic tracking problem of lower triangular systems is studied in [8] which can be viewed as a special case of the problem described in this paper in that the exogenous signal does not explicitly appear in plant which excludes the objective of disturbance rejection.

Typically, the output regulation problem is approached in two steps. In the first step, an appropriate dynamic compensator called internal model is found. Attaching the internal model to the given plant leads to an augmented system, and the internal model is such that the stabilization solution of the augmented system leads to the output regulation solution of the original plant. As a result, the second step will be centered on stabilizing the augmented system. When the exosystem is exactly known, the stabilization problem of the augmented system can often be handled by various robust control techniques. However, when the exosystem

The work of the first author was supported by the Australian Research Council under grant No. DP0878724. The work of the third author was supported by the Research Grants Council of the Hong Kong Special Administration Region under grant No. 412408.

Z. Chen is with the School of Electrical Engineering and Computer Science, The University of Newcastle, NSW 2308, Australia zhiyong.chen@newcastle.edu.au

L. Liu is with the Department of Information Physics and Computing, The University of Tokyo, Tokyo 113-8656, Japan lu_liu@ipc.i.u-tokyo.ac.jp

J. Huang is with the Department of Mechanical and Automation Engineering, The Chinese University of Hong Kong, Shatin, N.T., Hong Kong jhuang@mae.cuhk. edu.hk contains uncertain parameter, these uncertain parameters will enter the augmented systems and they cannot be handled by robust control techniques. Therefore, for this case, adaptive control technique has to be further introduced to handle this type of the unknown parameters. Compared with the work in [3] in which the semi-global output regulation problem is handled for the same class of systems by output feedback control, the main difficulty in the current problem is that we have to use the state feedback control to handle the global output regulation problem because the output feedback control cannot handle it without some additional restrictive assumptions. However, unlike the output feedback control where only one dynamic compensator corresponding to the input needs to be constructed, for the state feedback control case, we need to construct $r$ dynamic compensators corresponding to $r-1$ measurable states and one input where $r$ is the relative degree. As a result, the resulting augmented system will also be more complex. In particular, the adaptive control of the augmented system has to be constructed by a recursive approach. Each recursion necessitates a dynamic coordinate transformation which leads to a newly augmented system with more complex parameter uncertainty. This phenomenon is called "propagation of uncertainties". The approach in [8] can only handle the special case where the plant does not contain the exogenous signal, i.e., the exosystem accommodates only the tracking reference not the external disturbances. In this paper, by utilizing a newly developed robust adaptive control design approach in [9], we will extend the work in [8] to a more general output regulation problem described in Definition 2.1.

The rest of the paper is organized as follows. Section II provides a typical construction of an adaptive internal model to deal with uncertain exosystems. Based on the adaptive internal model and the robust adaptive control approach proposed in [9], the global robust output regulation problem formulated in this paper is solved. Then, a numerical example is given in Section III followed by some concluding remarks in Section IV.

\section{MAIN RESUlt}

To better reveal our idea and to avoid the notational complexity, in this paper we only focus on a special class of systems whose relative degree is two:

$$
\begin{aligned}
\dot{q}_{o} & =\kappa_{o}\left(q_{o}, q_{1}, v, w\right) \\
\dot{q}_{1} & =\kappa_{1}\left(q_{o}, q_{1}, v, w\right)+q_{2} \\
\dot{q}_{2} & =\varkappa_{2}\left(q_{o}, q_{1}, q_{2}, w\right)+\varkappa_{3}\left(q_{o}, q_{1}, v, w\right)+u \\
e & =q_{1}-q_{d}(v, w),
\end{aligned}
$$


where $q_{o} \in \mathbb{R}^{n_{o}}$ and $q_{1}, q_{2} \in \mathbb{R}$ are the states, $u(t) \in \mathbb{R}$ is the input, and $e(t) \in \mathbb{R}$ is the output representing the tracking error. The disturbance and/or reference signal $v \in \mathbb{R}^{q}$ is produced by an exosystem described by

$$
\dot{v}=A_{1}(\sigma) v, \quad v(0)=v_{o} .
$$

The unknown parameters $w \in \mathbb{R}^{p_{1}}$ and $\sigma \in \mathbb{R}^{p_{2}}$ are assumed to be in known compact sets. It is also assumed that all functions in the system (2.1) are polynomial. It should be noted that, in the system (2.1), we exclude the cross terms between $q_{2}$ and $v$ by using the functions $\varkappa_{2}$ and $\varkappa_{3}$ to facilitate the satisfaction of the linearly parameterization condition in applying the adaptive approach proposed in [9]. It is interesting to further develop a robust adaptive approach to deal with a more general nonlinear terms including such cross terms. Now, it is ready to give the precise formulation for the global output regulation problem as follows.

Definition 2.1: Global Output Regulation Problem: For given $\mathbb{V}, \mathbb{W}$, and $\mathbb{S}$, which are compact subsets of $\mathbb{R}^{q}, \mathbb{R}^{p_{1}}$, and $\mathbb{R}^{p_{2}}$ containing the origins, respectively, find a state feedback controller such that, for all $v(t) \in \mathbb{V}, w \in \mathbb{W}$, and $\sigma \in \mathbb{S}$, the trajectories of the closed-loop system, starting from any initial states, exist and are bounded for all $t>0$, and satisfy $\lim _{t \rightarrow \infty} e(t)=0$.

As mentioned in the introduction, we can handle the problem in two steps. The first step is to find the internal model to define the augmented system. To ascertain the existence of the desired internal model, we need the following two assumptions.

Assumption 2.1: For all $\sigma \in \mathbb{S}$, the exosystem is assumed to be neutrally stable in the sense that all the eigenvalues of $A_{1}(\sigma)$ are simple and have zero real part.

Assumption 2.2: There exists $\mathbf{q}_{o}(v, w, \sigma)$, a polynomial function in $v$ with coefficients depending on $w$ and $\sigma$ such that, for all $v \in \mathbb{V}, w \in \mathbb{W}$, and $\sigma \in \mathbb{S}$,

$$
\frac{\partial \mathbf{q}_{o}(v, w, \sigma)}{\partial v} A_{1}(\sigma) v=\kappa_{o}\left(\mathbf{q}_{o}(v, w, \sigma), q_{d}(v, w), v, w\right) .
$$

Remark 2.1: Assumption 2.1 means that the solution of the exosystem is a sum of finite many sinusoidal functions. Typically, $\sigma$ represents the frequencies of these sinusoidal functions. Under Assumption 2.2, we can define, for all $v \in$ $\mathbb{V}, w \in \mathbb{W}$, and $\sigma \in \mathbb{S}$,

$$
\begin{aligned}
\mathbf{q}_{1}(v, w)= & q_{d}(v, w) \\
\mathbf{q}_{2}(v, w, \sigma)= & \frac{\partial \mathbf{q}_{1}(v, w)}{\partial v} A_{1}(\sigma) v \\
& -\kappa_{1}\left(\mathbf{q}_{o}(v, w, \sigma), \mathbf{q}_{1}(v, w), v, w\right) \\
\mathbf{u}(v, w, \sigma)= & \frac{\partial \mathbf{q}_{2}(v, w, \sigma)}{\partial v} A_{1}(\sigma) v \\
& -\varkappa_{2}\left(\mathbf{q}_{o}(v, w, \sigma), \mathbf{q}_{1}(v, w), \mathbf{q}_{2}(v, w, \sigma), w\right) \\
& -\varkappa_{3}\left(\mathbf{q}_{o}(v, w, \sigma), \mathbf{q}_{1}(v, w), v, w\right) .
\end{aligned}
$$

which constitute the solution of the regulator equations for the system composed of (2.1) and (2.2) for all $v \in \mathbb{V}, w \in$
$\mathbb{W}$, and $\sigma \in \mathbb{S}$. And the solutions are polynomial in $v$ with coefficients depending on $w$ and $\sigma$.

Since $\mathbf{q}_{2}(v, w, \sigma)$ and $\mathbf{u}(v, w, \sigma)$ are polynomial in $v$ with coefficients depending on $w$ and $\sigma$, there exist integers $r_{1}$ and $r_{2}$, functions

$$
\begin{array}{r}
\vartheta_{i}(v, w, \sigma):=\operatorname{col}\left(\xi_{i}, \dot{\xi}_{i}, \cdots, \xi_{i}^{\left(r_{i}-1\right)}\right), i=1,2 \\
\xi_{1}=\mathbf{q}_{2}(v, w, \sigma), \xi_{2}=\mathbf{u}(v, w, \sigma),
\end{array}
$$

and matrices $\Phi_{i}(\sigma) \in \mathbb{R}^{r_{i} \times r_{i}}$ such that

$$
\dot{\vartheta}_{i}=\Phi_{i}(\sigma) \vartheta_{i}, \quad \xi_{i}=\left[\begin{array}{llll}
1 & 0 & \cdots & 0
\end{array}\right] \vartheta_{i} .
$$

Moreover, all the eigenvalues $\Phi_{i}(\sigma)$ are simple with zero real parts.

Next, pick any controllable pairs $\left(M_{i}, N_{i}\right)$ with $M_{i} \in$ $\mathbb{R}^{r_{i} \times r_{i}}, N_{i} \in \mathbb{R}^{r_{i} \times 1}$, and $M_{i}$ Hurwitz, and solve $T_{i}(\sigma)$ from the Sylvester equation

$$
T_{i}(\sigma) \Phi_{i}(\sigma)-M_{i} T_{i}(\sigma)=N_{i}\left[\begin{array}{llll}
1 & 0 & \cdots & 0
\end{array}\right]
$$

Let

$$
\begin{aligned}
\theta_{i}(v, w, \sigma) & :=T_{i}(\sigma) \vartheta_{i}(v, w, \sigma) \\
E_{i}(\sigma) & :=T_{i}(\sigma) \Phi_{i}(\sigma) T_{i}^{-1}(\sigma) \\
\Psi_{i}(\sigma) & :=\left[\begin{array}{llll}
1 & 0 & \cdots & 0
\end{array}\right] T_{i}^{-1}(\sigma)
\end{aligned}
$$

and

$$
\begin{aligned}
\theta(v, w, \sigma) & =\operatorname{col}\left(\theta_{1}(v, w, \sigma), \theta_{2}(v, w, \sigma)\right) \\
\alpha(\sigma, \theta) & =\operatorname{block} \operatorname{diag}\left(E_{1}(\sigma) \theta_{1}, E_{2}(\sigma) \theta_{2}\right) \\
\beta(\sigma, \theta) & =\operatorname{col}\left(\Psi_{1}(\sigma) \theta_{1}, \Psi_{2}(\sigma) \theta_{2}\right) \\
\mathrm{X} & =\operatorname{diag}\left(\mathrm{X}_{1}, \mathrm{X}_{2}\right), \mathbf{X}=M, N, T
\end{aligned}
$$

Then, it can be verified that $\{\theta(v, w, \sigma), \alpha(\sigma, \theta), \beta(\sigma, \theta)\}$ satisfies

$$
\begin{aligned}
\dot{\theta}(v, w, \sigma) & =\alpha(\sigma, \theta) \\
\xi & =\beta(\sigma, \theta) .
\end{aligned}
$$

The triplet $\{\theta(v, w, \sigma), \alpha(\sigma, \theta), \beta(\sigma, \theta)\}$ is called a steadystate generator of the system (2.1) and (2.2) with output $\operatorname{col}\left(q_{2}, u\right)$. Furthermore, let

$$
\begin{aligned}
\eta & :=\operatorname{col}\left(\eta_{1}, \eta_{2}\right) \\
\dot{\eta} & =M \eta+N \operatorname{col}\left(q_{2}, u\right)
\end{aligned}
$$

Then (2.4) defines the internal model for system (2.1) and (2.2) with output $\operatorname{col}\left(q_{2}, u\right)$. The composition of the system (2.1) and the internal model (2.4) is called an augmented system. If $\sigma$ is known, performing on the augmented system the following coordinate and input transformation

$$
\begin{aligned}
\bar{\eta}_{i} & =\eta_{i}-\theta_{i}-N_{i} \bar{q}_{i}, \quad i=1,2 \\
\bar{q}_{o} & =q_{o}-\mathbf{q}_{o}(v, w, \sigma) \\
\bar{q}_{1} & =e \\
\bar{q}_{2} & =q_{2}-\Psi_{1}(\sigma) \eta_{1} \\
\bar{u} & =u-\Psi_{2}(\sigma) \eta_{2}
\end{aligned}
$$


yields a system of the following form

$$
\begin{aligned}
\dot{\bar{q}}_{0} & =\bar{\kappa}_{o}\left(\overline{\mathcal{Q}}_{1}, d\right) \\
\dot{\bar{\eta}}_{i} & =M_{i} \bar{\eta}_{i}+\gamma_{i}\left(\bar{\zeta}_{i-1}, \mathcal{Q}_{i}, d\right) \\
\dot{\bar{q}}_{i} & =\bar{\kappa}_{i}\left(\overline{\mathcal{Q}}_{i}, d\right)+\bar{q}_{i+1}, i=1,2
\end{aligned}
$$

where $d=\operatorname{col}(v, w), \quad \overline{\mathcal{Q}}_{1}:=\operatorname{col}\left(\bar{q}_{o}, \bar{q}_{1}\right), \quad \overline{\mathcal{Q}}_{2} \quad:=$ $\operatorname{col}\left(\bar{q}_{o}, \bar{q}_{1}, \bar{q}_{2}\right), \bar{\zeta}_{1}:=\bar{\eta}_{1}, \bar{\zeta}_{2}:=\operatorname{col}\left(\bar{\eta}_{1}, \bar{\eta}_{2}\right), \bar{\kappa}_{i}(0, d)=0$, and $\gamma(0,0, d)=0$. If there is a controller of the form

$$
\bar{u}=g\left(\lambda, \overline{\mathcal{Q}}_{2}\right), \dot{\lambda}=\psi\left(\lambda, \overline{\mathcal{Q}}_{2}\right)
$$

that solve the global stabilization problem of the system (2.6), then the following controller

$$
\begin{aligned}
u & =g\left(\lambda, \overline{\mathcal{Q}}_{2}\right)+\Psi_{2}(\sigma) \eta_{2} \\
\dot{\lambda} & =\psi\left(\lambda, \overline{\mathcal{Q}}_{2}\right) \\
\dot{\eta} & =M \eta+N \operatorname{col}\left(q_{2}, u\right)
\end{aligned}
$$

solves the output regulation problem of the original system (2.1) [6]. Nevertheless, when $\sigma$ is unknown, the controller (2.7) is not implementable. To overcome the difficulty caused by the uncertain exosystem, we consider the following coordinate transform:

$$
\begin{aligned}
z_{i} & =\eta_{i}-\theta_{i}-N_{i} x_{i}, i=1,2 \\
x_{o} & =q_{o}-\mathbf{q}_{o}(v, w, \sigma) \\
x_{1} & =e \\
x_{2} & =q_{2} \\
d & =\operatorname{col}(v, w, \sigma), \mu=\operatorname{col}(w, \sigma) .
\end{aligned}
$$

Now, we can define a variety of measurement outputs as follows:

$$
\begin{aligned}
& \bar{y}_{1}=\operatorname{col}\left(e, q_{o}, q_{1}\right) \\
& y_{1}=\operatorname{col}\left(e, q_{o}, q_{1}, \eta_{1}\right) \\
& \bar{y}_{2}=\operatorname{col}\left(e, q_{o}, q_{1}, q_{2}, \eta_{1}\right) \\
& y_{2}=\operatorname{col}\left(e, q_{o}, q_{1}, q_{2}, \eta_{1}, \eta_{2}\right) .
\end{aligned}
$$

The measurement outputs will be used in the feedback to controller. Under the new coordinates, the augmented system (2.1) and (2.4) can be rewritten as follows:

$$
\begin{aligned}
\dot{x}_{o} & =f_{o}\left(x_{o}, x_{1}, d\right) \\
\dot{z}_{1} & =M_{1} z_{1}+\gamma_{1}\left(\chi_{1}, d\right) \\
\dot{x}_{1} & =f_{1}\left(\zeta_{1}, \chi_{1}, d\right)+l_{1}\left(y_{1}, \mu\right)+x_{2}, \\
\dot{z}_{2} & =M_{2} z_{2}+\gamma_{2}\left(\zeta_{1}, \chi_{2}, d\right)+h_{2}\left(\bar{y}_{2}, \mu\right) \\
\dot{x}_{2} & =f_{2}\left(\zeta_{2}, \chi_{2}, d\right)+l_{2}\left(y_{2}, \mu\right)+u
\end{aligned}
$$

with $\chi_{1}:=\operatorname{col}\left(x_{o}, x_{1}\right), \chi_{2}:=\operatorname{col}\left(x_{o}, x_{1}, x_{2}\right), \zeta_{1}:=z_{1}$ and $\zeta_{2}:=\operatorname{col}\left(z_{1}, z_{2}\right)$. The functions are defined as follows:

$$
\begin{aligned}
f_{o}\left(x_{o}, x_{1}, d\right)= & \kappa_{o}\left(q_{o}, q_{1}, v, w\right)-\kappa_{o}\left(\mathbf{q}_{o}, \mathbf{q}_{1}, v, w\right) \\
\gamma_{1}\left(\chi_{1}, d\right)= & M_{1} N_{1} x_{1}-N_{1} A_{1} \\
f_{1}\left(\zeta_{1}, \chi_{1}, d\right)= & A_{1}+\Psi_{1}(\sigma) \eta_{1}-\Psi_{1}(\sigma) \theta_{1} \\
\gamma_{2}\left(\zeta_{1}, \chi_{2}, d\right)= & M_{2} N_{2} x_{2}-N_{2} A_{2}+N_{2} \Psi_{1}(\sigma) \\
& \times E_{1}(\sigma)\left(N_{1} x_{1}+z_{1}\right) \\
f_{2}\left(\zeta_{2}, \chi_{2}, d\right)= & A_{2}-\Psi_{1}(\sigma) E_{1}(\sigma)\left(N_{1} x_{1}+z_{1}\right) \\
& +\Psi_{2}(\sigma)\left(N_{2} x_{2}+z_{2}\right)
\end{aligned}
$$

and

$$
\begin{aligned}
l_{1}\left(y_{1}, \mu\right) & =-\Psi_{1}(\sigma) \eta_{1} \\
h_{2}\left(\bar{y}_{2}, \mu\right) & =-N_{2} B-N_{2} \Psi_{1}(\sigma) E_{1}(\sigma) \eta_{1} \\
l_{2}\left(y_{2}, \mu\right) & =B+\Psi_{1}(\sigma) E_{1}(\sigma) \eta_{1}-\Psi_{2}(\sigma) \eta_{2}
\end{aligned}
$$

where

$$
\begin{aligned}
A_{1}:= & \kappa_{1}\left(q_{o}, q_{1}, v, w\right)-\kappa_{1}\left(\mathbf{q}_{o}, \mathbf{q}_{1}, v, w\right) \\
A_{2}:= & \varkappa_{2}\left(q_{o}, q_{1}, \Psi_{1}(\sigma) \eta_{1}, w\right)-\varkappa_{2}\left(\mathbf{q}_{o}, \mathbf{q}_{1}, \mathbf{q}_{2}, w\right) \\
& +\varkappa_{3}\left(q_{o}, q_{1}, v, w\right)-\varkappa_{3}\left(\mathbf{q}_{o}, \mathbf{q}_{1}, v, w\right) \\
B= & \varkappa_{2}\left(q_{o}, q_{1}, q_{2}, w\right)-\varkappa_{2}\left(q_{o}, q_{1}, \Psi_{1}(\sigma) \eta_{1}, w\right) .
\end{aligned}
$$

Having derived the augmented system which has the property that $f_{i}(0,0, d)=0$ for $i=0,1,2$, and $\gamma_{i}(0,0, d)=0$ for $i=1,2$, we will proceed to the second step, i.e., to globally stabilize the augmented system (2.8) in the sense the states of the closed-loop are globally bounded and $\lim _{t \rightarrow \infty} e(t)=0$. It can be seen that the solution of such a problem implies that of the output regulation problem for the original system (2.1. The augmented system (2.8) contains both dynamic uncertainty and static uncertainty and the stabilization of such a system has never been studied until recently by the authors in [9]. In what follows, we will apply the approach in [9] to solve the stabilization problem of the augmented system (2.8).

To summarize the main result, we introduce one more assumption.

Assumption 2.3: There exists a sufficiently smooth function $V\left(q_{o}\right)$ bounded by some class $\mathcal{K}_{\infty}$ polynomial functions, such that, along the trajectories of $\dot{q}_{o}=\kappa_{o}\left(q_{o}, q_{1}, v, w\right)$,

$$
\frac{d V\left(q_{o}\right)}{d t} \leq-\left\|q_{o}\right\|^{2}+\pi\left(q_{1}\right)
$$

for some polynomial positive definite function $\pi$.

Theorem 2.1: Under Assumptions 2.1 and 2.2 and 2.3, the global robust output regulation problem of (2.1) is solvable.

Proof: We will explicitly construct the controller in this proof. To this end, let's define some notations using the algorithm developed in [9].

First, as seen in [10] or [9], there exist polynomial functions $\alpha_{i}(\cdot), i=1,2$, such that, under the new coordinate

$$
\tilde{x}_{o}=x_{o}, \tilde{x}_{1}=x_{1}, \tilde{x}_{2}=x_{2}-\alpha_{1}\left(\tilde{x}_{1}\right), \tilde{x}_{3}=x_{3}-\alpha_{2}\left(\tilde{x}_{2}\right),
$$

the system (2.8) with $h_{i}=0$ and $l_{i}=0$ becomes

$$
\begin{aligned}
\dot{\tilde{x}}_{0} & =f_{0}\left(\tilde{x}_{o}, \tilde{x}_{1}, d\right) \\
\dot{z}_{i} & =M_{i} z_{i}+\varphi_{i}\left(\zeta_{i-1}, \tilde{\chi}_{i}, d\right) \\
\dot{\tilde{x}}_{i} & =\phi_{i}\left(\zeta_{i}, \tilde{\chi}_{i}, d\right)+\alpha_{i}\left(\tilde{x}_{i}\right)+\tilde{x}_{i+1}, i=1,2(2.10)
\end{aligned}
$$

for some polynomial functions $\varphi_{i}$ and $\phi_{i}$. Moreover, there exist positive definite and radially unbounded functions $V\left(\zeta_{2}\right)$ and $W\left(\tilde{\chi}_{2}\right)=\sum_{i=0}^{2} W_{i}\left(\tilde{x}_{i}\right)$ with $\tilde{\chi}_{2}:=$ $\operatorname{col}\left(\tilde{x}_{o}, \tilde{x}_{1}, \tilde{x}_{2}\right)$, such that, along the trajectories of the system (2.10),

$$
\frac{d\left(V\left(\zeta_{2}\right)+W\left(\tilde{\chi}_{2}\right)\right)}{d t} \leq-k\left(\zeta_{2}, \tilde{\chi}_{2}\right)
$$


for some positive definite function $k(\cdot, \cdot)$.

Next, we define the notations

$$
\left(\bar{\chi}_{i}\right)-\left(\varrho_{i}, \varpi_{i}\right)-\left(\bar{\zeta}_{i}, \xi_{i}\right)-\left(\rho_{i}, \omega_{i}\right)-\left(\psi_{i}\right)-\left(\lambda_{i}\right)
$$

for $i=1$. In particular, Let

$$
\ell_{1}\left(y_{1}, \mu\right)=l_{1}\left(y_{1}, \mu\right)=-\Psi_{1}(\sigma) \eta_{1},
$$

and

$$
\Psi_{1}(\sigma)=\omega_{1}^{\top}(\mu) \bar{\Psi}_{1}
$$

for some column function vector $\omega_{1}$ and a constant $\bar{\Psi}_{1}$. Then,

$$
\ell_{1}\left(y_{1}, \mu\right)=\rho_{1}\left(y_{1}\right) \omega_{1}(\mu)
$$

for

$$
\rho_{1}\left(y_{1}\right)=-\eta_{1}^{\top} \bar{\Psi}_{1}^{\top}
$$

Let $\hat{\omega}_{1}$ be a vector with the same dimension of $\omega_{1}$ and

$$
\psi_{1}\left(y_{1}\right)=k_{1}\left(d W_{1}\left(x_{1}\right) / d x_{1}\right) \rho_{1}^{\top}\left(y_{1}\right)
$$

for any $k_{1}>0$. The vector $\hat{\omega}_{1}$ is governed by $\dot{\hat{\omega}}_{1}=\psi_{1}\left(y_{1}\right)$. Now, it is ready to see (2.12) has been defined for $i=1$ with

$$
\begin{array}{r}
\bar{\chi}_{1}=\operatorname{col}\left(x_{o}, \bar{x}_{1}\right), \bar{x}_{1}=x_{1}, \varrho \in \mathbb{R}^{o}, \varpi_{1} \in \mathbb{R}^{o}, \\
\bar{\zeta}_{1}=\bar{z}_{1}=z_{1}, \xi_{1}=s_{1} \in \mathbb{R}^{0}, \lambda_{1}=\hat{\omega}_{1} .
\end{array}
$$

For $i=2$ the notations (2.12) are defined in the following order.

- $\bar{\chi}_{2}:=\operatorname{col}\left(x_{o}, \bar{x}_{1}, \bar{x}_{2}\right)$ where

$$
\bar{x}_{2}=x_{2}+\rho_{1}\left(y_{1}\right) \hat{\omega}_{1}-\alpha_{1}\left(\bar{x}_{1}\right) .
$$

- Define

$$
\begin{aligned}
& \hbar_{2}\left(\bar{y}_{2}, \hat{\omega}_{1}, \mu\right) \\
= & \gamma_{2}\left(\zeta_{1}, \chi_{2}, d\right)-\gamma_{2}\left(\bar{\zeta}_{1}, x_{o}, \bar{x}_{1}, \bar{x}_{2}+\alpha_{1}\left(\bar{x}_{1}\right), d\right) \\
& +h_{2}\left(\bar{y}_{2}, \mu\right) \\
= & -M_{2} N_{2} \rho_{1}\left(y_{1}\right) \hat{\omega}_{1}+N_{2} \varkappa_{2}\left(q_{o}, q_{1}, q_{2}, w\right) \\
& -N_{2} \varkappa_{2}\left(q_{o}, q_{1}, \Psi_{1}(\sigma) \eta_{1}, w\right)-N_{2} \Psi_{1}(\sigma) E_{1}(\sigma) \eta_{1} .
\end{aligned}
$$

Since all functions are in polynomial form, the function $\hbar_{2}\left(\bar{y}_{2}, \hat{\omega}_{1}, \mu\right)$ satisfies the following linearly parameterization property:

$$
\hbar_{2}\left(\bar{y}_{2}, \hat{\omega}_{1}, \mu\right)=\varrho_{2}\left(\bar{y}_{2}, \lambda_{1}\right) \varpi_{2}(\mu)
$$

for a sufficiently smooth function matrix $\varrho_{2}$ and a column function vector $\varpi_{2}$.

- Let $\bar{\zeta}_{2}:=\operatorname{col}\left(\bar{z}_{1}, \bar{z}_{2}\right)$ and $\xi_{2}:=\operatorname{col}\left(s_{1}, s_{2}\right)$ where $s_{2}$ is a square matrix governed by

$$
\dot{s}_{2}=M_{2} s_{2}+\varrho_{2}\left(\bar{y}_{2}, \lambda_{1}\right)
$$

and $\bar{z}_{2}=z_{2}-s_{2} \varpi_{2}(\mu)$.

- The derivative of $\rho_{1}\left(y_{1}\right)$ is

$$
\bar{\rho}_{1}\left(y_{2}, \mu\right)=-\dot{\eta}_{1}^{\top} \bar{\Psi}_{1}^{\top}=-\left(M_{1} \eta_{1}+N_{1} x_{2}\right)^{\top} \bar{\Psi}_{1}^{\top} .
$$

Let

$$
\begin{aligned}
& \ell_{2}\left(y_{2}, \xi_{2}, \hat{\omega}_{1}, \mu\right) \\
= & f_{2}\left(\zeta_{2}, \chi_{2}, d\right)-f_{2}\left(\bar{\zeta}_{2}, x_{o}, \bar{x}_{1}, \bar{x}_{2}+\alpha_{1}\left(\bar{x}_{1}\right), d\right) \\
& +l_{2}\left(y_{2}, \mu\right)+\left(\partial \alpha_{1}\left(\bar{x}_{1}\right) / \partial \bar{x}_{1}\right) \rho_{1}\left(y_{1}\right) \tilde{\omega}_{1}(\mu) \\
& +\bar{\rho}_{1}\left(y_{2}, \mu\right) \hat{\omega}_{1}(\mu)+\rho_{1}\left(y_{1}\right) \psi_{1}\left(y_{1}\right) \\
= & -\Psi_{2}(\sigma) N_{2} \rho_{1}\left(y_{1}\right) \hat{\omega}_{1}+\varkappa_{2}\left(q_{o}, q_{1}, q_{2}, w\right) \\
& -\varkappa_{2}\left(q_{o}, q_{1}, \Psi_{1}(\sigma) \eta_{1}, w\right)+\Psi_{2}(\sigma) s_{2} \varpi_{2}(\mu) \\
& +l_{2}\left(y_{2}, \mu\right)+\left(\partial \alpha_{1}\left(\bar{x}_{1}\right) / \partial \bar{x}_{1}\right) \rho_{1}\left(y_{1}\right) \tilde{\omega}_{1}(\mu) \\
& +\bar{\rho}_{1}\left(y_{2}, \mu\right) \hat{\omega}_{1}(\mu)+\rho_{1}\left(y_{1}\right) \psi_{1}\left(y_{1}\right) .
\end{aligned}
$$

Clearly, the function $\ell_{2}\left(y_{2}, \xi_{2}, \hat{\omega}_{1}, \mu\right)$ is linearly parameterized in the sense that

$$
\ell_{2}\left(y_{2}, \xi_{2}, \hat{\omega}_{1}, \mu\right)=\rho_{2}\left(y_{2}, \xi_{2}, \lambda_{1}\right) \omega_{2}(\mu)
$$

for a sufficiently smooth row vector function $\rho_{2}$ and a column function vector $\omega_{2}$.

- For any $k_{2}>0$, let

$$
\psi_{2}\left(y_{2}, \xi_{2}, \lambda_{1}\right)=k_{2}\left(d W_{2}\left(\bar{x}_{2}\right) / d \bar{x}_{2}\right) \rho_{2}^{\top}\left(y_{2}, \xi_{2}, \lambda_{1}\right) .
$$

- Let $\lambda_{2}:=\operatorname{col}\left(\hat{\omega}_{1}, \hat{\omega}_{2}\right)$, where $\hat{\omega}_{1}$ is vector variable governed by $\dot{\hat{\omega}}_{2}=\psi_{2}\left(y_{2}, \xi_{2}, \lambda_{1}\right)$.

With the above notations, it has been proved in [9] that, the system (2.8) becomes

$$
\begin{aligned}
\dot{x}_{o}= & f_{o}\left(x_{o}, x_{1}, d\right) \\
\dot{\bar{z}}_{i}= & M_{i} \bar{z}_{i}+\varphi_{i}\left(\bar{\zeta}_{i-1}, \bar{\chi}_{i}, d\right) \\
\dot{\bar{x}}_{i}= & \phi_{i}\left(\bar{\zeta}_{i}, \bar{\chi}_{i}, d\right)+\alpha_{i}\left(\bar{x}_{i}\right)-\rho_{i}\left(y_{i}, \xi_{i}, \lambda_{i-1}\right) \tilde{\omega}_{i}+\bar{x}_{i+1} \\
& i=1,2 .
\end{aligned}
$$

with $\bar{x}_{3}=u+\rho_{2}\left(y_{2}, \xi_{2}, \lambda_{2-1}\right) \hat{\omega}_{2}-\alpha_{2}\left(\bar{x}_{2}\right)$. The system (2.14) can also be derived by a direct calculation. Moreover, it has been proved in [9] that, with $\bar{x}_{3}=0$, i.e.,

$$
u=-\rho_{2}\left(y_{2}, \xi_{2}, \lambda_{2-1}\right) \hat{\omega}_{2}+\alpha_{2}\left(\bar{x}_{2}\right),
$$

the global robust regulation for the system (2.8) is solved. The proof is thus completed.

Remark 2.2: Moreover, it can be shown that of

$$
\begin{gathered}
\lim _{t \rightarrow 0} \eta_{i}(t)-\theta_{i}(t)=0, i=1,2 \\
\lim _{t \rightarrow 0} q_{i}(t)-\mathbf{q}_{i}(v(t), w, \sigma)=0, i=0,1,2 \\
\lim _{t \rightarrow 0} u(t)-\mathbf{u}(v(t), w, \sigma)=0 .
\end{gathered}
$$

Thus the global servomechanism problem with an unknown exosystem is solved in the same sense as the case where the exosystem is known even though, in our problem formulation, the requirements (2.16) to (2.18) are not explicitly specified.

\section{AN EXAMPLE}

We consider the global robust output regulation problem of the system (2.1) with the following parameters:

$$
\begin{aligned}
\dot{q}_{o} & =-q_{o}+0.2 e \\
\dot{q}_{1} & =0.5 q_{o} \sin q_{1}+w_{1} q_{1}+q_{2} \\
\dot{q}_{2} & =w_{2} q_{o}^{2}+w_{3} q_{1}+u \\
e & =q_{1}-v_{1}
\end{aligned}
$$


coupled with an exosystem

$$
\begin{aligned}
& \dot{v}_{1}=-\sigma v_{2} \\
& \dot{v}_{2}=\sigma v_{1} .
\end{aligned}
$$

These equations formulate the control problem of designing a state-feedback regulator to have the output $q_{1}$ of system (2.19) asymptotically tracks a sinusoidal signal of unknown frequency with arbitrarily large fixed amplitude, produced by exosystem (2.20), in the presence of three uncertain parameters $\left(w_{1}, w_{2}, w_{3}\right)$.

Since Assumptions 2.1, 2.2 and 2.3 are all satisfied, the global robust output regulation is solvable. To explicitly give the controller, we note that the regulator equations associated with (2.19) and (2.20) have a globally defined solution in polynomial form as follows

$$
\begin{aligned}
\mathbf{q}_{o}(v, w, \sigma) & =0 \\
\mathbf{q}_{1}(v, w, \sigma) & =v_{1} \\
\mathbf{q}_{2}(v, w, \sigma) & =-\sigma v_{2}-w_{1} v_{1} \\
\mathbf{u}(v, w, \sigma) & =-\sigma^{2} v_{1}+w_{1} \sigma v_{2}-w_{3} v_{1} .
\end{aligned}
$$

To construct the internal model, we have the matrices

$$
\Phi_{1}(\sigma)=\Phi_{2}(\sigma)=\left[\begin{array}{cc}
0 & 1 \\
-\sigma^{2} & 0
\end{array}\right]
$$

and

$$
M_{1}=M_{2}=\left[\begin{array}{cc}
-1 & 0 \\
0 & -2
\end{array}\right], N_{1}=N_{2}=\left[\begin{array}{l}
0.2 \\
0.5
\end{array}\right] \text {. }
$$

Next, we can solve $T_{1}(\sigma)$ and $T_{2}(\sigma)$ from the Sylvester equation as

$$
T_{1}(\sigma)=T_{2}(\sigma)=\left[\begin{array}{cc}
\frac{0.2}{\sigma^{2}+1} & -\frac{0.2}{\sigma^{2}+1} \\
\frac{1}{\sigma^{2}+4} & -\frac{0.5}{\sigma^{2}+4}
\end{array}\right],
$$

hence,

$$
\Psi_{1}(\sigma)=\Psi_{2}(\sigma)=\left[\begin{array}{ll}
-5 \sigma^{2}-5 & 2 \sigma^{2}+8
\end{array}\right] .
$$

After the introduction of the internal model (2.4), we can convert the system into (2.8). Next, we will give the detailed calculation on the quantities used in the controller design. In particular, we can choose

$$
\alpha_{1}\left(\tilde{x}_{1}\right)=-K_{1} \tilde{x}_{1}, \alpha_{2}\left(\tilde{x}_{2}\right)=-K_{2} \tilde{x}_{2}\left(1+\tilde{x}_{2}^{2}\right)
$$

and

$$
W_{1}\left(\tilde{x}_{1}\right)=\left(\tilde{x}_{1}^{2}+\tilde{x}_{1}^{4}\right) / 2, W_{2}\left(\tilde{x}_{2}\right)=3 \tilde{x}_{2}^{2} / 2
$$

where $K_{1}$ and $K_{2}$ are determined by the bound of $w_{1}, w_{2}, w_{3}$ and $\sigma$.

Since $\ell_{1}\left(y_{1}, \mu\right)=-\Psi_{1}(\sigma) \eta_{1}$, we have

$$
\rho_{1}\left(y_{1}\right)=-\eta_{1}^{\top} \bar{\Psi}_{1}^{\top}, \bar{\Psi}_{1}=\left[\begin{array}{ll}
-5 & 2 \\
-5 & 8
\end{array}\right], \omega_{1}(\mu)=\operatorname{col}\left(\sigma^{2}, 1\right) .
$$

Next, note $\Psi_{1}(\sigma) E_{1}(\sigma)=\left[-10 \sigma^{2}-10,2 \sigma^{2}+8\right]$. Since

$$
\hbar_{2}\left(\bar{y}_{2}, \hat{\omega}_{1}, \mu\right)=M_{2} N_{2} \eta_{1}^{\top} \bar{\Psi}_{1}^{\top} \hat{\omega}_{1}-N_{2} \Psi_{1}(\sigma) E_{1}(\sigma) \eta_{1},
$$

we have

$$
\begin{aligned}
\varrho_{2}\left(\bar{y}_{2}, \hat{\omega}_{1}\right) & =\left[\begin{array}{ll}
0 & M_{2} N_{2} \eta_{1}^{\top} \bar{\Psi}_{1}^{\top} \hat{\omega}_{1}
\end{array}\right]-N_{2} \eta_{1}^{\top} \hat{\Psi}_{1}^{\top}, \\
\hat{\Psi}_{1} & =\left[\begin{array}{ll}
-10 & 2 \\
-10 & 8
\end{array}\right], \varpi_{2}(\mu)=\operatorname{col}\left(\sigma^{2}, 1\right) .
\end{aligned}
$$

We note the derivative of $\rho_{1}\left(y_{1}\right)$ is

$$
\bar{\rho}_{1}\left(y_{2}, \mu\right)=-\dot{\eta}_{1}^{\top} \bar{\Psi}_{1}^{\top}=-\left(M_{1} \eta_{1}+N_{1} x_{2}\right)^{\top} \bar{\Psi}_{1}^{\top},
$$

which is well defined and is available to the control law. A calculation shows

$$
\begin{aligned}
\ell_{2}\left(y_{2}, \xi_{2}, \hat{\omega}_{1}, \mu\right)= & {\left[A_{11} A_{12}+A_{21} A_{22}\right] \operatorname{col}\left(\sigma^{4}, \sigma^{2}, 1\right) } \\
& +B \operatorname{col}\left(\sigma^{2}, 1\right)+C
\end{aligned}
$$

with

$$
\begin{aligned}
A= & \bar{\Psi}_{1} s_{2} \\
B= & -\left(N_{2} \rho_{1}\left(y_{1}\right) \hat{\omega}_{1}\right)^{\top} \bar{\Psi}_{1}^{\top}+\eta_{1}^{\top} \hat{\Psi}_{1}^{\top}-\eta_{2}^{\top} \bar{\Psi}_{1}^{\top} \\
& -\left(\partial \alpha_{1}\left(\bar{x}_{1}\right) / \partial \bar{x}_{1}\right) \rho_{1}\left(y_{1}\right) \\
C= & \left(\partial \alpha_{1}\left(\bar{x}_{1}\right) / \partial \bar{x}_{1}\right) \rho_{1}\left(y_{1}\right) \hat{\omega}_{1} \\
& +\bar{\rho}_{1}\left(y_{2}, \mu\right) \hat{\omega}_{1}+\rho_{1}\left(y_{1}\right) \psi_{1}\left(y_{1}\right) .
\end{aligned}
$$

Therefore, we have

$$
\begin{aligned}
\rho_{2}\left(y_{2}, \xi_{2}, \hat{\omega}_{1}\right)= & {\left[\begin{array}{ll}
0 & B
\end{array}\right] } \\
& +\left[\begin{array}{lll}
A_{11} & A_{12}+A_{21} & C+A_{22}
\end{array}\right] \\
\omega_{2}= & \operatorname{col}\left(\sigma^{4}, \sigma^{2}, 1\right) .
\end{aligned}
$$

The simulation is conducted with the parameters $\sigma=$ $0.2, w_{1}=-0.4, w_{2}=0.8, w_{3}=0.3, v_{1}(0)=10, v_{2}(0)=$ $0, q_{o}(0)=5, q_{1}(0)=8, q_{2}(0)=-1$, and the initial values of the remaining states being zero.

With the adaptive controller proposed in this paper, the tracking error quickly converges to zero. The performance is plotted in Figures 1 and 2 for plant states, input, and internal model states. We also observe the convergence of the parameter estimation in the simulation. Due to the overparametrization, the unknown frequency $\sigma$ is estimated three times in terms of $\hat{\sigma}_{1}:=\sqrt{\hat{\omega}_{11}}, \hat{\sigma}_{2}:=\sqrt[4]{\hat{\omega}_{21}}$, and $\hat{\sigma}_{3}:=$ $\sqrt{\hat{\omega}_{22}}$. The convergence is plotted in Figure 3 .

\section{CONCLUSION}

In this paper, we have presented the solvability conditions for the global adaptive robust output regulation for a class of lower triangular systems subject to uncertain exosystems. The result can be viewed as an application of the recently developed integrated robust and adaptive approach [9]. An example has been used to illustrate the controller design procedure with the simulation.

\section{REFERENCES}

[1] A. Isidori and C. I. Byrnes. Output regulation of nonlinear systems. IEEE Transactions on Automatic Control, 35:131-140, 1990.

[2] H. Khalil. Robust servomechanism output feedback controllers for feedback linearizable systems. Automatica, 30:1587-1589, 1994.

[3] A. Serrani, A. Isidori, and L. Marconi. Semiglobal nonlinear output regulation with adaptive internal model. IEEE Transactions on Automatic Control, 46:1178-1194, 2001. 
[4] C. I. Byrnes and A. Isidori. Limit sets, zero dynamics and internal models in the problem of nonlinear output regulation. IEEE Transactions on Automatic Control, 48:1712-1723, 2003.

[5] Z. Ding. Universal disturbance rejection for nonlinear systems in output feedback form. IEEE Transactions on Automatic Control, 48:1222-1226, 2003.

[6] J. Huang and Z. Chen. A general framework for tackling the output regulation problem. IEEE Transactions on Automatic Control, 49:2203-2218, 2004.

[7] X. D. Ye and J. Huang. Decentralized adaptive output regulation for a class of large-scale nonlinear systems. IEEE Transactions on Automatic Control, 48:276-281, 2003.

[8] Z. Chen and J. Huang. Global tracking of uncertain nonlinear cascaded systems with adaptive internal model. Proceedings of the 41st IEEE Conference on Decision and Control, pages 3855-3862, 2002.

[9] Z. Chen and J. Huang. Robust adaptive regulation of polynomial systems with dynamic uncertainties. Proceedings of the 48st IEEE Conference on Decision and Control, to appear.

[10] Z. Chen and J. Huang. A Lyapunov's direct method for the global robust stabilization of nonlinear cascaded systems. Automatica, 44:745-752, 2008.
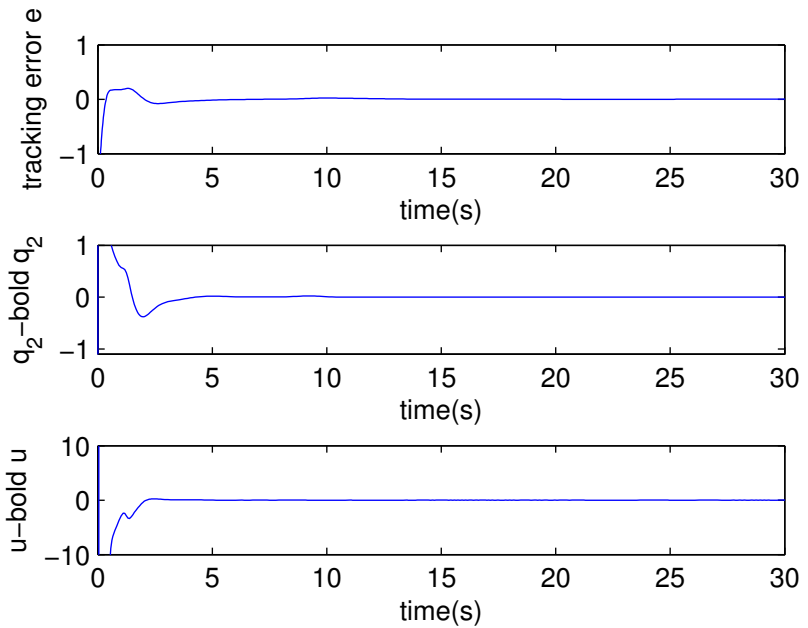

Fig. 1. Profile of the tracking errors for the plant states and input
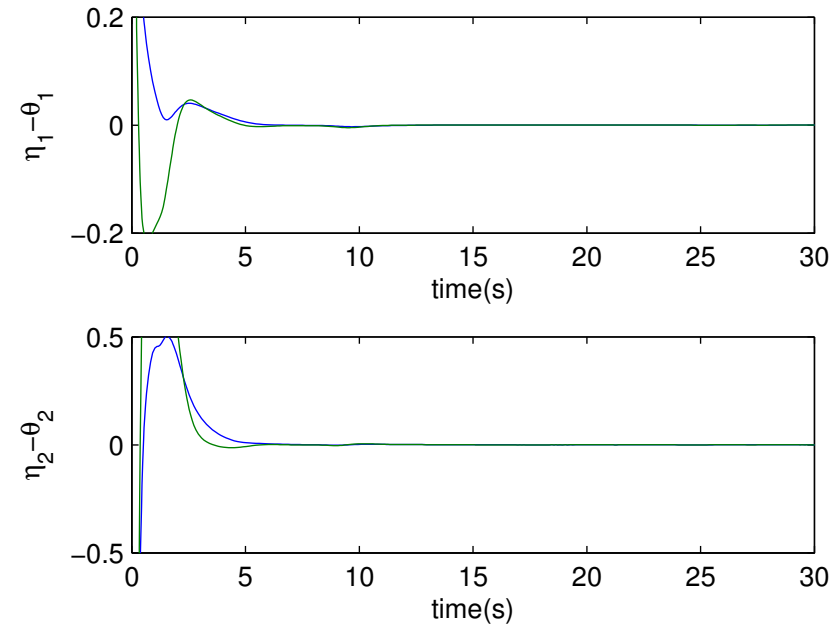

Fig. 2. Profile of the tracking errors for the internal model states
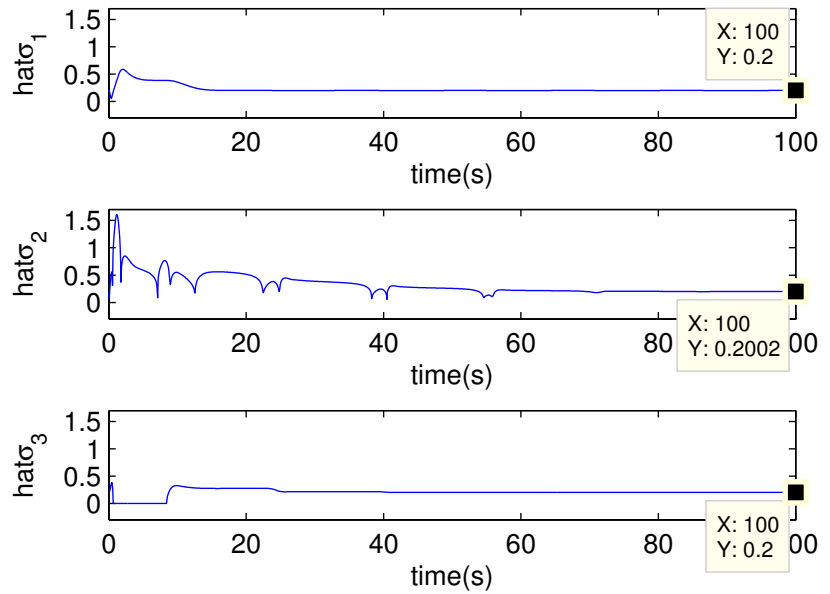

Fig. 3. Profile of the estimated frequencies. 